\title{
Dispositif pédagogique en médecine palliative visant le développement de la réflexivité chez des étudiants. Utilisation d'un portfolio électronique
}

\author{
An educational device used in palliative medicine to develop students' \\ reflexive thinking abilities. Use of an electronic portfolio
}

\author{
Didier GIET ${ }^{1,4}$, Valérie MASSART ${ }^{1}$, Mélanie DEUM ${ }^{2}$ et Jean-François BRICHANT ${ }^{3}$ \\ ${ }^{1}$ Département de médecine générale (DUMG), Université de Liège, Liège, Belgique \\ ${ }^{2}$ Service Guidance-Étude, Administration de l'enseignement et des étudiants, Université de Liège, Liège, Belgique \\ ${ }^{3}$ Centre hospitalier universitaire de Liège, Département d'anesthésie-réanimation, Université de Liège, Liège, Belgique \\ ${ }^{4}$ IFRES-ULg Institut de Formation et de Recherche en Enseignement Supérieur, Université de Liège, Liège, Belgique
}

Manuscrit reçu le 6 juillet 2011 ; commentaires éditoriaux formulés aux auteurs le 21 août 2011 et le 12 février 2012 ; accepté pour publication le 16 février 2012

\begin{abstract}
Mots-clés
Formation médicale ; réflexivité ; portfolio électronique ; tutorat ; médecine palliative
\end{abstract}

Keywords Medical education; reflexive thinking; electronic portfolio; tutor; palliative care
Résumé - Contexte : Le cours de médecine palliative est dispensé aux étudiants en avant-dernière année des études médicales de $2^{\mathrm{e}}$ cycle par deux enseignants, un médecin généraliste et un médecin hospitalier. Ce cours est dispensé depuis 10 ans selon un format traditionnel magistral. De nouveaux objectifs et de nouvelles modalités pédagogiques ont été introduits en 20102011. Ce dispositif concerne 95 étudiants. But : Cet article a pour objet de décrire le dispositif pédagogique du cours d'introduction à la médecine palliative et de fin de vie. Matériel et méthodes : L'objectif principal du dispositif pédagogique est l'apprentissage réflexif des étudiants. Les outils pédagogiques sont le portfolio électronique, une vignette clinique évolutive, l'accompagnement à distance de chaque étudiant par un tuteur, des cours pluridisciplinaires en présentiel. Résultats : Le dispositif a eu lieu comme prévu. Tous les étudiants ont ouvert et complété leur portfolio. Conclusions et perspectives : Le dispositif pédagogique sera reconduit l'année académique prochaine en tenant compte des résultats de l'évaluation certificative des portfolios et de l'analyse du dispositif par les étudiants, tuteurs et enseignants.

Abstract - Context: Students take the palliative medicine course in the year preceding their last year of medical studies. The two teachers, a general practitioner and a hospital-based physician, have been giving the course in a traditional lecture format for 10 years. New goals and new teaching methods were introduced in 2010-2011. The device involved 95 students. Purpose: The article aims to describe the educational device used in the palliative medicine and end-of-life course. Material and methods: The device is mainly designed to enhance students' reflexive learning. Teaching tools include the electronic portfolio, clinical vignettes (case reports), remote tutor support for all students and multidisciplinary courses held in a 
classroom setting. Results: The device was used according to plan. Ninety-nine percent of students accessed and completed their portfolio. Conclusions: The educational approach will be repeated in the next academic year and take into account results of the portfolio certification evaluation as well as the analysis of the device by students, tutors and teachers.

\section{Contexte}

L'avant-dernière année des études médicales de deuxième cycle à l'Université de Liège est constituée entièrement de stages en milieux hospitalier et ambulatoire (alors que les deux années précédentes sont constituées de un à deux jours de stage d'observation par semaine) et de deux journées de cours par mois à l'Université. Au cours de ces journées, un cours d'introduction à la «médecine palliative et de fin de vie » est dispensé. Il a été mis sur pied il y a 10 ans par deux enseignants titulaires, un professeur, médecin généraliste, et un professeur médecin hospitalier.

Les années antérieures, ce cours était dispensé de façon magistrale par de multiples intervenants de diverses disciplines et validé uniquement sur base de la présence au cours. Une démarche d'évaluation du cours a été entamée en raison d'un amphithéâtre qui se vidait au fil des séances, bien que le cours soit obligatoire. Les étudiants jugeaient les interventions trop théoriques et juxtaposées, loin des questions et préoccupations qu'ils rencontrent notamment en stage. De nombreuses redites étaient observées. En conséquence, les étudiants se détournaient de la formation proposée dans le domaine médecine palliative et de fin de vie.

Ce cours devant faire l'objet d'une remise de notes (exigence académique), le dispositif de formation et d'évaluation des étudiants a été remis en question. Actuellement, les étudiants en troisième année de master sont au nombre de 95.

L'objectif visé par le nouveau dispositif pédagogique est de susciter une attitude réflexive chez les étudiants, centrée sur l' analyse, le jugement et la régulation, trois facettes de la métacognition ${ }^{[1]}$ mais aussi de prendre conscience et de réfléchir à des thématiques spécifiques liées à la médecine palliative et de fin de vie.
D'autres objectifs plus spécifiques sont également poursuivis par ce dispositif qui tend à susciter davantage d'interactions entre les encadrants et les étudiants en mettant en œuvre des méthodologies plus participatives. C'est dans cette optique que le portfolio et le tutorat qui y est associé ont été pensés. Se centrer sur l'étudiant et sur son cheminement dans ses apprentissages est au centre de cette innovation pédagogique.

\section{Cadre conceptuel du dispositif pédagogique}

\author{
La perspective socio-cognitiviste et socio- \\ constructiviste
}

Le paradigme épistémologique général dans laquelle s'inscrit le dispositif pédagogique rapporté se réfère aux développements contemporains des sciences de l'apprentissage et notamment aux perspectives socio-cognitiviste et socio-constructiviste et aux contributions théoriques d'Ausubel ${ }^{[2]}$, Vygotsky ${ }^{[3]}$ et Bandura ${ }^{[4]}$.

Cette perspective «postule fondamentalement que l'apprentissage est une activité intentionnelle de traitement de l'information et de construction de sens, que les étudiants construisent graduellement leurs connaissances à partir de ce qu'ils savent déjà et que les contextes d'enseignement et d'apprentissage, au sein desquels les interactions sociales jouent un rôle essentiel, conditionnent la transférabilité des connaissances » ${ }^{[5]}$.

On peut résumer la perspective « socio-constructiviste et interactive » en trois volets ${ }^{[6]}$ : un volet lié à la dimension constructiviste, un volet lié aux interactions sociales, un volet lié aux interactions avec le 
Tableau I. Principes de l'approche pédagogique centrée sur l'étudiant. D'après l'American Psychological Association ${ }^{[11]}$.

\begin{tabular}{l}
\hline $\begin{array}{l}\text { L'apprentissage est plus efficace quand il s'agit d'un processus intentionnel de construction de sens à partir de } \\
\text { l'expérience et d'informations nouvelles. }\end{array}$ \\
\hline Le caractère stratégique de l'apprentissage implique que l'étudiant soit orienté par des buts pertinents. \\
\hline $\begin{array}{l}\text { L'apprenant efficient est capable d'établir de multiples liens entre les informations nouvelles et ses connaissances } \\
\text { antérieures. }\end{array}$ \\
\hline $\begin{array}{l}\text { L'apprenant efficient est capable de créer et d'utiliser un répertoire de stratégies de pensée et de raisonnement } \\
\text { adaptes à la complexité de ses buts d'apprentissage. }\end{array}$ \\
\hline $\begin{array}{l}\text { Le recours à des stratégies d'ordre supérieur pour contrôler et gérer ses opérations mentales favorise la pensée } \\
\text { critique et créative. }\end{array}$ \\
\hline $\begin{array}{l}\text { L'apprentissage est influencé par des facteurs environnementaux, dont font partie la culture, l'environnement } \\
\text { technologique et les pratiques éducatives. }\end{array}$ \\
\hline $\begin{array}{l}\text { La motivation à apprendre est influencée par les états émotionnels, les croyances, les intérêts et les buts de } \\
\text { l'apprenant ainsi que par ses habitudes de pensée. }\end{array}$ \\
\hline $\begin{array}{l}\text { La motivation à apprendre est favorisée par l'exposition à des tâches de difficulté adaptée, cohérentes avec les } \\
\text { intérêts personnels de l'apprenant, sur lesquels il peut exercer un contrôle. }\end{array}$ \\
\hline $\begin{array}{l}\text { L'engagement et la persévérance dans des activités d'apprentissage complexes sont stimulés par la motivation et } \\
\text { peuvent être favorisés par l'enseignant. }\end{array}$ \\
\hline $\begin{array}{l}\text { L'apprentissage est plus efficace quand sont prises respectivement en compte les occasions favorables ou les } \\
\text { contraintes liées au développement physique, intellectuel, affectif et social de l'apprenant. }\end{array}$ \\
\hline L'apprentissage est influencé par les interactions sociales et interpersonnelles de l'apprenant. \\
\hline $\begin{array}{l}\text { Les apprenants développent des stratégies d'apprentissage influencées à la fois par des aptitudes constitutionnelles } \\
\text { et par leurs expériences antérieures. }\end{array}$ \\
\hline $\begin{array}{l}\text { L'apprentissage est plus efficient quand les caractéristiques individuelles, culturelles, linguistiques et sociales de } \\
\text { l'apprenant sont prises en compte. }\end{array}$ \\
\hline $\begin{array}{l}\text { La détermination d'objectifs adaptés mais défiants et l'évaluation diagnostique du processus et des résultats de } \\
\text { l'apprentissage font partie intégrante du processus d'apprentissage. }\end{array}$ \\
\hline
\end{tabular}

milieu, la dimension constructiviste postulant pour sa part que le sujet construit ses connaissances à travers sa propre activité et que l'objet manipulé au cours de cette activité n'est autre que sa propre connaissance. Ce double postulat suppose donc de la part du sujet qui construit ses connaissances une activité réflexive sur ses propres connaissances.

Dans le cadre de dispositifs de formation professionnelle qui se réclament de cet ancrage conceptuel et qui sont mis en œuvre authentiquement en cohérence avec lui, l'étudiant est amené à prendre une posture professionnelle où il doit poser en permanence un jugement sur ses propres compétences et lacunes, afin de proposer une intervention adaptée ${ }^{[7]}$. Au fur et à mesure de l'apprentissage, l'étudiant est amené également à affiner et préciser les stratégies de résolution de problèmes développées. Les nouveaux apprentissages construits sont donc au service de ces régulations et sont réintégrés dans les schémas d'actions des étudiants ${ }^{[8-10]}$.

L'American Psychological Association ${ }^{[11]}$ a décrit 14 principes pour guider l'action pédagogique dans une perspective socio-constructiviste. Ces principes, présentés dans le tableau I, doivent être pris en compte dans une approche pédagogique centrée sur l'étudiant.

La pratique réflexive

Selon Perrenoud ${ }^{[12]}$, la clef de la professionnalisation est la pratique réflexive. La capacité réflexive est considérée par de nombreux auteurs comme une caractéristique essentielle de la compétence professionnelle ${ }^{[11]}$. 
L'expertise professionnelle se caractérise notamment par la capacité du praticien à faire face à des situations complexes, qui partagent plusieurs caractéristiques : les indices nécessaires à leur résolution ne sont pas tous disponibles d'emblée, leur solution n'est pas standardisée, la nature du problème à résoudre évolue en cours d'investigation, les « personnes ressources » sont souvent en désaccord quant à la meilleure solution à mettre en œuvre, etc. ${ }^{[13-15]}$.

Leclercq ${ }^{[1]}$ définit la métacognition comme étant l'ensemble des « jugements, analyses et/ou régulations observables, effectués par l'apprenant sur ses propres performances (processus ou produits d'apprentissage), ceci dans des situations pré, per ou post performance ». Nous nous basons sur cette définition mais avons plus particulièrement ciblé trois dimensions : jugement, analyse et régulation.

Selon cet auteur ${ }^{[1]}$, les jugements sont des évaluations effectuées par l'apprenant lui-même du degré de qualité qu'il attribue à sa performance et/ou à sa compétence (par exemple par anticipation de la performance si la performance n'a pas encore eu lieu). Les analyses sont les justifications et explications (les « pourquoi »), que l'apprenant élabore quant à la qualité de sa performance, ainsi que les «attributions causales » faites au sujet de ce qui lui arrive. Les régulations sont les reconductions ou changements dans les méthodes de travail, dans les habitudes, dans l'environnement, les décisions, etc. Elles correspondent à ce que Gombert appelle « les capacités qu'un individu a de contrôler et planifier ses propres processus cognitifs en vue de la réalisation d'un but ou d'un objectif déterminé ».

De nombreux enseignements peuvent aussi être retenus de la revue systématique de Mann ${ }^{[17]}$ lorsque l'on veut mettre en place un dispositif pédagogique visant le développement de la réflexivité. Nous retenons les principes suivants :

- la réflexivité peut être utile lorsqu'elle est considérée comme une stratégie d'apprentissage. Elle peut alors aider les apprenants à intégrer de nouveaux apprentissages et à les connecter avec leurs compétences existantes.

- Elle peut également aider les apprenants à prendre en compte, à expliciter et intégrer les aspects affectifs de leurs apprentissages.
- La réflexivité n'est pas un processus évident pour les étudiants. Le plus souvent, il est d'ailleurs tacite chez les praticiens chevronnés. Une piste est que les enseignants rendent eux-mêmes explicites leurs processus de réflexivité. Le travail pluridisciplinaire peut s'en trouver également favorisé si la pratique réflexive est collaborative.

- Surtout en début d'apprentissage, les étudiants peuvent avoir besoin d'une structure pour guider cette activité réflexive. Ils peuvent demander une rétroaction sur le contenu et le processus de leur réflexion. Il s'agit alors d'une occasion d'examiner les forces et faiblesses et de déterminer les besoins d'apprentissage.

- La réflexivité peut être un outil d'auto-évaluation encourageant les étudiants à améliorer leur jugement.

- La supervision et l'accompagnement des étudiants sont essentiels dans le développement d'une pratique réflexive.

- La réflexivité doit faire partie de la culture d'enseignement et d'apprentissage. Elle doit être valorisée et légitimée.

L'ensemble de ces principes répondent aux facteurs de motivation décrits par Viau ${ }^{[18]}$. En effet, en partant directement des connaissances préalables des étudiants et en les soutenant dans l'auto-évaluation de leurs propres compétences, les formateurs mettent l'accent davantage sur la progression de l'étudiant par rapport à lui-même que par rapport à une norme. L'étudiant est donc plus à même de cerner le développement de ses compétences et la construction de ses savoirs. De plus, le dispositif place l'étudiant au centre de sa formation en lui donnant la possibilité de piloter ses apprentissages, de les réguler, de mettre en avant ses acquis, de pallier ses lacunes et d'avoir des répercussions sur le cours théorique en formulant des questions à l'égard des enseignants. Enfin, ce genre de dispositif fait grandement sens pour les étudiants car il se base principalement sur leur propre progression et est intégré dans un système d'évaluation et de formation authentiques, proche des cas qu'ils rencontreront dans leur vie professionnelle future. En valorisant les tâches demandées, en laissant une certaine autonomie aux étudiants et en leur donnant les clés de leur réussite en main ainsi qu'en valorisant leurs 
La patiente est de plus en plus fatiguée. Les douleurs sont à présent continues, insupportables malgré les traitements administrés. La patiente demande à être réhospitalisée. Le bilan a montré une progression rapide de la maladie au niveau osseux et l'apparition de métastases pulmonaires. La patiente considère que son cas est « dépassé » et demande l'interruption des traitements à visée étiologique.

Comment allez-vous passer des soins curatifs aux soins palliatifs? Comment organisez-vous les soins palliatifs à l'hôpital ?

En collaboration avec le médecin traitant et la famille, une équipe de soins supportifs est mise en place au domicile de la patiente.

Des membres de l'équipe de soins jugent la situation insupportable et s'accordent difficilement sur l'attitude à adopter.

Comment organisez-vous les soins palliatifs au domicile de la patiente?

Fig. 1. Exemple d'une des séquences de la vignette clinique.

compétences, ce dispositif novateur influence également positivement la motivation des étudiants.

Le contexte pédagogique, dont l'utilisation du portfolio $^{[7,19]}$, la narration de l'étudiant au moyen de ce portfolio ${ }^{[17]}$, l'aspect électronique de l'outil ${ }^{[21,22]}$ et la distance de l' accompagnement des tuteurs ${ }^{[23]}$ qui sont d'autant de modalités particulières, influencent considérablement l'apprentissage de l'étudiant.

Cet article a pour objet de décrire le dispositif pédagogique du cours d'introduction à la médecine palliative et de fin de vie, élaboré en lien avec le cadre conceptuel présenté précédemment.

\section{Méthodes}

Un outil d'apprentissage et de réflexivité : le portfolio électronique

Chaque étudiant est invité à ouvrir un portfolio électronique personnel. Ici, le portfolio est considéré notamment comme un moyen d'interactions à distance entre l'étudiant et un tuteur dont les tâches sont décrites ci-dessous. L'étudiant va y consigner ses connaissances antérieures, ses expériences, ses questions, ses réflexions, auxquelles le tuteur va réagir.

Le portfolio va également permettre de garder une trace de la réflexion des étudiants au fil de l'analyse de l'histoire clinique d'un patient mais aussi être une trace des apprentissages des étudiants en matière de médecine palliative et de fin de vie.
Le portfolio est aussi un outil d'auto-évaluation pour l'étudiant. Des grilles mensuelles et des grilles à compléter à mi-parcours et en fin de dispositif sont fournies à l'étudiant pour faire le point sur le regard qu'il porte sur ses apprentissages.

Le portfolio électronique, sur base de critères et d'indicateurs définis et connus des étudiants, sera évalué par les enseignants et une note est attribuée à chacun d'eux.

\section{Ressources didactiques}

\section{Utilisation d'une vignette clinique évolutive}

Le dispositif que nous présentons repose sur l'utilisation d'une vignette évolutive pour contextualiser l'apprentissage. Une vignette clinique présente une histoire médicale d'un patient réel ou imaginé (Figure 1). Scindée en cinq séquences, elle décrit l'histoire de la maladie et son évolution mais également le contexte global et holistique du patient. Les étudiants sont ainsi amenés à analyser la vignette selon un canevas qui leur est proposé dans leur portfolio et à déterminer quelle serait leur prise en charge du patient.

\section{Guides d'accompagnement}

Des supports pédagogiques sous forme de guides d'accompagnement ont été rédigés pour soutenir le travail des étudiants mais aussi celui des différents enseignants. Ces guides présentent les objectifs et les 
outils utilisés dans ce dispositif, le calendrier et les échéances de travail ainsi que la description des rubriques à compléter par les étudiants dans un portfolio. Les outils d'auto-évaluation y sont également développés et justifiés, ainsi que la grille d'évaluation.

\section{Cours théoriques}

Le dispositif prévoit également des cours théoriques. Chaque mois, un cours de deux heures est dispensé à l'ensemble des 95 étudiants en un seul grand groupe par l'un des intervenants de différentes disciplines. Il a été élaboré en fonction des objectifs spécifiques du dispositif, chaque fois en lien avec une séquence de la vignette clinique. Ce cours est préparé par les divers intervenants (médecins spécialistes, médecins généralistes, psychologue,...) en fonction de questions suscitées par la lecture de la vignette clinique, préalablement envoyées par les étudiants via le portfolio. Le cours est ainsi adapté aux préoccupations et aux soucis des étudiants, ce qui implique que les intervenants acceptent de revoir leur enseignement quelques jours avant le cours, afin de mieux répondre aux questions des étudiants.

Rôles et stratégies d'enseignement et d'accompagnement

\section{Tutorat}

Un tuteur, investi professionnellement dans le domaine, encadre un groupe de huit étudiants. Il rencontre les étudiants une première fois pour entamer l'analyse de la première séquence de vignette. Il accompagnera ensuite chaque étudiant personnellement, à distance, via un portfolio électronique.

Concrètement, il est proposé au tuteur de renvoyer des commentaires, des feed-backs en réaction aux écrits des étudiants. L'objectif n'est pas de fournir des informations aux étudiants mais de susciter et approfondir leur réflexion, de les inviter à se poser des questions : «penses-tu que... ? » « as-tu réfléchi au domaine... ? », «que penses-tu de...», « et si le patient disait ça..., que penses-tu utile de faire »?

Il est aussi suggéré que le tuteur invite l'étudiant à décontextualiser, à aller plus loin que le cas décrit.
Son rôle est de permettre à l'étudiant de repérer ses points forts et ses faiblesses, de l'aider à progresser dans sa réflexion et ses apprentissages et d'interroger la pertinence de ses interventions. Le tuteur n'est pas amené à juger ni à évaluer l'étudiant (il ne remettra pas de note).

\section{Evaluation des portfolios}

L'évaluation des portfolios par les enseignants fera l'objet d'une publication ultérieure. Elle a été effectuée par les deux enseignants titulaires à l'aide d'une grille d'évaluation comportant des critères et des indicateurs (tableau II), qui seront éventuellement reformulés à la lumière de cette première expérience.

\section{Dispositif de formation}

Le cours «Médecine palliative et de fin de vie» s'étend sur six mois, de septembre à février à raison de $13 \mathrm{~h} 30$ en présentiel à l'université. S'y ajoutent des travaux à domicile. Une première séance de deux heures est consacrée à l'introduction du dispositif, en grand groupe, pour une présentation des objectifs, des outils, de l'évaluation.

La vignette clinique proposée présente l'évolution du patient. Elle va permettre aux étudiants de réfléchir aux différents aspects de la prise en charge exigée pour le cas décrit. Il est prévu que cette réflexion s'accompagne d'interactions entre l'étudiant et son tuteur au travers du portfolio, d'un moment d'enseignement en grand groupe axé sur les savoirs utiles à la compréhension de la vignette pour une étape donnée et de pistes d'écriture réflexive permettant aux étudiants de faire le point sur leur niveau de maîtrise des savoirs et sur leurs compétences.

La figure 2 illustre les étapes du dispositif pédagogique. La vignette clinique est divisée en cinq séquences et est révélée étape après étape aux étudiants. Chacune des parties aborde des objectifs spécifiques de la médecine palliative et de fin de vie. Le portfolio est donc structuré en cinq parties correspondant chacune à un moment de l'histoire clinique du patient. Pour chaque séquence de l'histoire clinique du patient, les étudiants répondent à plusieurs 
Tableau II. Grille d'évaluation des portfolios des étudiants par les deux enseignants responsables.

\begin{tabular}{|c|c|c|}
\hline $\begin{array}{l}\text { Rubriques du portfolio } \\
\text { complétées par } \\
\text { l'étudiant }\end{array}$ & Critères & $\begin{array}{c}\text { Indicateurs } \\
\text { et nombre de points }\end{array}$ \\
\hline $\begin{array}{l}\text { «e que je sais } \\
\text { déjà », « Mes } \\
\text { questions » et « Ma } \\
\text { prise en charge } \\
\text { actuelle » }\end{array}$ & $\begin{array}{l}\text { Engagement dans la } \\
\text { tâche } \\
\text { Densité du contenu } \\
\text { Structure/forme }\end{array}$ & $\begin{array}{l}0 \text { = L'étudiant n'a pas complété son portfolio. } \\
1 \text { = L'étudiant a partiellement complété son portfolio. } \\
2 \text { = Le portfolio de l'étudiant est complet, son investissement est } \\
\text { visible. }\end{array}$ \\
\hline $\begin{array}{l}\text { «Ce que je ferais } \\
\text { autrement » }\end{array}$ & $\begin{array}{l}\text { Capacité réflexive } \\
\text { (pertinence de l'analyse } \\
\text { et de la régulation de la } \\
\text { situation décrite dans la } \\
\text { vignette) }\end{array}$ & $\begin{array}{l}0 \text { = L'étudiant n'identifie aucun savoir, savoir-faire, savoir-être. } \\
1 \text { = L'étudiant identifie des thématiques de cours et en fait une } \\
\text { sorte de listing. } \\
2 \text { ou } 3 \text { = L'étudiant porte une réflexion sur les thématiques du } \\
\text { cours et les contenus abordés, sur ce que cela lui a apporté. D'une } \\
\text { manière générale, il prend du recul par rapport aux savoirs appris. } \\
2 \text { ou } 3 \text { = L'étudiant propose une régulation de ses pratiques } \\
\text { (reconduction ou changement). } \\
4=\text { L'étudiant justifie et argumente les raisons de la régulation de } \\
\text { son action. }\end{array}$ \\
\hline $\begin{array}{l}\text { Fiches d'auto- } \\
\text { évaluation mensuelle }\end{array}$ & $\begin{array}{l}\text { Qualité de l'auto- } \\
\text { évaluation et } \\
\text { identification des } \\
\text { éléments déclencheurs de } \\
\text { progrès }\end{array}$ & $\begin{array}{l}0 \text { = l'étudiant ne s'est pas autoévalué à chaque étape. } \\
2 \text { = L'étudiant rempli les autoévaluations du portfolio en } \\
\text { identifiant uniquement que des contenus/thématiques de cours } \\
\text { dans son argumentation. } \\
3 \text { = L'étudiant rempli les autoévaluations du portfolio en faisant } \\
\text { preuve d'introspection, de prise de distance, d'implication, de } \\
\text { réflexivité, de lien avec ses connaissances/expériences (l'étudiant } \\
\text { se positionne en parlant en « je » professionnel c.-à-d. en se } \\
\text { projetant dans la peau d'un professionnel). L'argumentation porte } \\
\text { sur des compétences personnelles et professionnelles. }\end{array}$ \\
\hline $\begin{array}{l}\text { Fiche de bilan des } \\
\text { apprentissages à mi- } \\
\text { parcours et en fin de } \\
\text { dispositif }\end{array}$ & $\begin{array}{l}\text { Degré d'apprentissage } \\
\text { (Bilan d'atteinte des } \\
\text { objectifs fixés par le } \\
\text { cours et décrits) }\end{array}$ & $\begin{array}{l}0 \text { = L'étudiant n'identifie pas précisément ses apprentissages/ses } \\
\text { lacunes et ne les explique pas. } \\
2 \text { = L'étudiant a complété uniquement le bilan de ses } \\
\text { apprentissages (croix). } \\
+1 \text { ou }+2=\text { SI l'étudiant justifie son niveau d'acquisition sur le } \\
\text { plan de ses compétences (pas d'évaluation des contenus du cours). } \\
+1 \text { ou }+2=\text { SI l'étudiant propose des pistes d'action en lien avec } \\
\text { son développement professionnel (et non la régulation du cours). }\end{array}$ \\
\hline
\end{tabular}

questions décrites ci-dessous. Chaque rubrique tend à mobiliser chez les étudiants une des facettes de la pratique réflexive à savoir le jugement, l'analyse et la régulation.

\section{Étape 1. Explicitation des connaissances et de l'expérience}

Une des premières activités demandées aux étudiants face à l'histoire clinique est d'exprimer leurs connaissances au sujet des concepts qui y sont abordés. Il leur est aussi demandé d'exprimer leur expérience éventuelle dans ces domaines en répondant à la rubrique «ce que je sais déjà » ou «quelles sont actuellement mes connaissances, mes expériences?». Elle sollicite la mémoire déclarative, sémantique mais aussi épisodique mais aussi la capacité d'analyse, une des facettes de la réflexivité.

Pour aider les étudiants à compléter le portfolio, il leur est proposé quelques questions-guides telles 


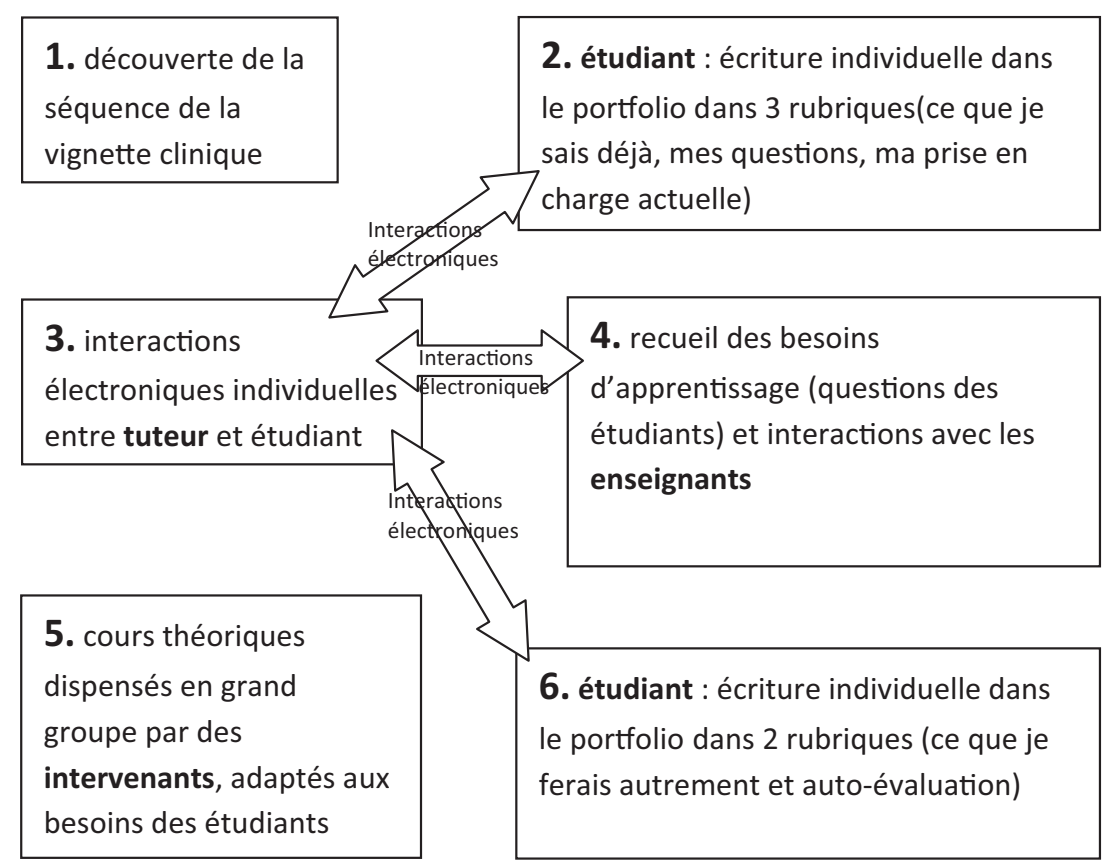

Fig. 2. Étapes du dispositif pédagogique.

que par exemple : « Face à la situation médicale d'un patient, quelles sont les connaissances que vous réactivez ? »ou encore : «Quel est votre expérience, votre vécu relatifs à ce type de situation?»; «À quelles situations déjà rencontrées pensez-vous ? »

\section{Étape 2. Formulation de questions}

L'analyse de la vignette clinique suppose chez l'étudiant un travail d'auto-évaluation et de jugement de ses connaissances et le tri entre ce qu'il maîtrise et ses lacunes, pour une prise en charge optimale du patient illustrée dans la vignette et de son entourage. L'étudiant est invité à formuler des questions afin de répondre à ses besoins d'apprentissage dans la rubrique «mes questions » de son portfolio.

L'étudiant formule des questions à propos des domaines qui lui sont méconnus, peu connus ou pas suffisamment précis pour réinvestir ses connaissances dans la prise en charge d'un patient. Ces questions sont lues par le tuteur sur le portfolio des étudiants qu'il encadre. Ce dernier les synthétise et les fait parvenir aux enseignants qui doivent préparer le cours théorique.

\section{Étape 3. Description de l'action prévue}

Il est demandé aux étudiants de se projeter en tant que médecin du patient décrit dans la vignette. Après analyse de la situation, les étudiants doivent tracer les grandes lignes de leurs actions dans la rubrique « ma prise en charge actuelle ». C'est la capacité de jugement, une autre facette de la réflexivité, qui est sollicitée. Les questions-guides proposées aux étudiants sont par exemple «Que pensez-vous qu'il serait utile de faire et dans quel ordre ? », « Quels propos tiendrez-vous auprès du patient, auprès de son entourage, auprès des acteurs médicaux et para-médicaux ? ». Les tuteurs recueillent à ce stade toutes les questions formulées par les étudiants ainsi que les domaines « connus » des étudiants et les transmettent aux intervenants. Ceux-ci adaptent leur enseignement à ces éléments. Chaque cours est construit autour de thématiques déterminées, en lien direct avec les faits décrits dans la vignette.

\section{Étape 4. Régulation de l'action envisagée}

Lorsque les étudiants ont assisté au cours, ils sont amenés à intégrer les nouvelles données dans leur 
prise en charge du patient au sein de la rubrique « ce que je ferais autrement ». C'est la capacité de régulation, une autre facette de la réflexivité, qui est sollicitée.

Les étudiants réinvestissent ces nouveaux éléments appris dans la prise en charge de la situation palliative selon quelques questions-guides comme : « À présent, comment prendriez-vous en charge la situation ?»; «En quoi serait-elle différente de ce que vous évoquiez à l'étape 1 ? »

\section{Étape 5. Auto-évaluation}

Après chaque séquence, les étudiants sont amenés à évaluer la progression de leurs apprentissages au moyen de deux outils. Le premier est une grille d'auto-évaluation mensuelle (figure 3) qui tend à recueillir, mois après mois, la perception de l'étudiant sur sa progression dans le domaine des soins palliatifs. Mais c'est surtout l'argumentation qu'il en fait, qui est discutée entre le tuteur et l'étudiant.

Le second outil d'auto-évaluation est utilisé à deux reprises, à mi-parcours et en fin de dispositif. L'étudiant est amené à établir le bilan de ses apprentissages, en prenant appui sur la liste des objectifs « matière » déterminés pour ce cours. Une grille d'auto-évaluation est proposée (figure 4). Ce sont essentiellement les rubriques « justification du niveau d'acquisition » et les « perspectives » qui font l'objet d'échanges entre tuteurs et étudiants.

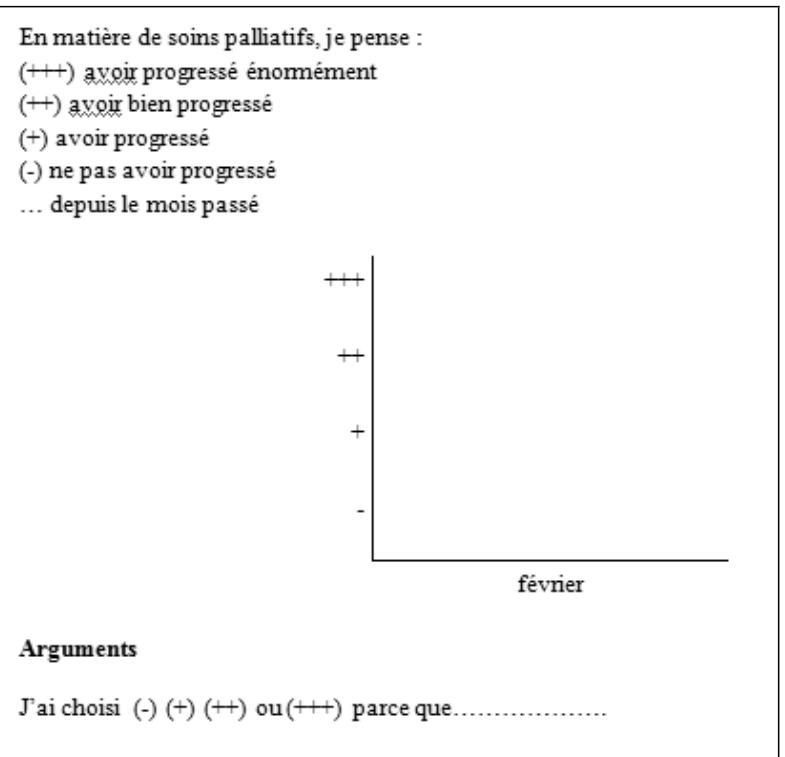

Fig. 3. Grille d'auto-évaluation mensuelle.

\section{Résultats}

Les 95 étudiants ont ouvert leur portfolio. Tous l'ont complété, même si certaines rubriques restent parfois vides. Ces étudiants ont été accompagnés par 13 tuteurs, chacun prenant en charge 7 à 8 étudiants.

Les tuteurs ont accompagné un groupe d'étudiants à distance au moyen du portfolio et du courrier électronique. Les questions des étudiants ont été recueilles au terme de chaque phase du dispositif et

\begin{tabular}{|c|c|c|c|c|c|c|c|c|}
\hline \multicolumn{9}{|c|}{ A mi-parcours, quel est le regard que je porte sur mes apprentissages, l'évolution de ceux-ci ? } \\
\hline & \multirow[t]{2}{*}{ Objectifs d'apprentissage } & \multirow{2}{*}{$\begin{array}{c}\text { Non } \\
\text { acquis }\end{array}$} & \multicolumn{3}{|c|}{ En voie d'acquisition } & \multirow[t]{2}{*}{ acquis } & \multirow{2}{*}{$\begin{array}{c}\text { Justifications du niveau } \\
\text { d'acquisition }\end{array}$} & \multirow[t]{2}{*}{ Perspectives } \\
\hline & & & + & ++ & +++ & & & \\
\hline & - annonce d'une mauvaise nouvelle & & & & & & & \\
\hline & $\begin{array}{l}\text { - traitements à visée palliative et à visée } \\
\text { curatives: l'intention thérapeutique }\end{array}$ & & & & & & & \\
\hline & - dynamique familiale en situation de crise & & & & & & & \\
\hline & - délimitation des soins & & & & & & & \\
\hline & - déclaration anticipée d'euthanasie & & & & & & & \\
\hline & $\begin{array}{l}\text { - abord du vécu du malade et de sa } \\
\text { famille }\end{array}$ & & & & & & & \\
\hline & - traitements des effets indésirables de la & & & & & & & \\
\hline
\end{tabular}

Fig. 4. Extrait de la grille d'auto-évaluation à mi-parcours et en fin de dispositif sur la base des objectifs d'apprentissage. 
ont été transmises aux intervenants. L'ensemble des enseignements ont été dispensés avec adaptation préalable du contenu. Une grande majorité des étudiants ont participé aux enseignements magistraux, alors que les années précédentes, le cours était déserté.

Les deux enseignants titulaires ont évalué chaque portfolio d'étudiants sur base d'une grille critériée. Une discussion entre les deux enseignants s'en est suivie pour établir la note définitive de l'étudiant. Les premières évaluations montrent que de nombreux étudiants confondent auto-évaluation et évaluation des enseignements. En effet, beaucoup jugent les cours auxquels ils ont assisté pour décrire leurs acquis et la progression de ceux-ci ( J'ai beaucoup appris de l'intervention du Professeur en matière de...», « Le Professeur auraient dû insister davantage sur la notion...»).

Les évaluations des portfolios montrent le plus souvent des ébauches de pratique réflexive des étudiants : cette capacité ne fait pas l'objet d'encouragement ni d'évaluation explicite dans le cursus médical de base. Il est dès lors nécessaire d'accompagner ces démarches réflexives et de soutenir les étudiants dans leur questionnement. Toutefois, certaines réflexions concernant les soins palliatifs sont parfois très approfondies et pertinentes.

\section{Discussion}

Les principes du socio-constructivisme, les caractéristiques de la capacité réflexive et les conditions de la motivation de l'étudiant trouvent une application dans le présent dispositif. Les forces et faiblesses du dispositif et de la démarche sont également présentées ici.

Les forces du dispositif

\section{Apprendre dans une perspective socio-constructiviste}

Donner du sens aux apprentissages ${ }^{[5,11]}$ est rendu possible par l'utilisation d'une histoire clinique proche de la réalité. Cette histoire est longitudinale, à l'image de la pratique professionnelle. Le dispositif de formation est authentique, proche des démarches à mettre en œuvre dans l'exercice du métier. Il permet à tous les étudiants d'être confrontés simultanément à une même situation palliative (ce qui n'est jamais le cas sur le terrain de stage) et de mener une réflexion, quels que soit leurs acquis antérieurs. Le bagage des étudiants est en effet très variable en termes d'expériences personnelles ou professionnelles, les parcours de stage n'étant pas semblables.

Dans le dispositif proposé, l'activité est également centrée sur la propre progression de l'étudiant. Il est donc au centre du dispositif, ce qui, aux yeux des étudiants, lui confère une valeur particulière.

Les interactions sociales, centrales dans le socioconstructivisme $^{[5]}$, sont présentes dans les échanges entre tuteur et étudiant, entre étudiants, et entre étudiants et enseignants.

«L'implantation d'un portfolio, par les conceptions pédagogiques qu'elle implique, constitue souvent une rupture par rapport aux pratiques antérieures des étudiants et des enseignants, qu'il ne faut pas sous-estimer » ${ }^{[24]}$. Les étudiants ont en effet été confrontés dans ce dispositif à un paradigme centré sur les apprenants qui leur est peu familier : il leur était demandé d'émettre, par écrit ${ }^{[20]}$, leur prérequis, leurs connaissances, d'évaluer leur progression, leurs apprentissages alors qu'ils sont plutôt habitués à écouter les enseignants et à attendre que les enseignants les évaluent. Il y a véritablement rupture avec le modèle classique d'apprentissage frontal et de restitution du savoir.

\section{Susciter la motivation}

Un contrôle sur la tâche ${ }^{[11]}$ est offert aux étudiants par l'adaptation des cours magistraux suite aux questions préalablement posées. Ils ont donc la possibilité d'influencer le déroulement du cours et d'augmenter la cohérence entre les contenus abordés et leurs propres niveaux de connaissance. De plus, l'évaluation formative et continue, portant sur la progression et sur la réflexivité des étudiants, procure des feedbacks réguliers sur leurs cheminements en mettant en lumière forces et lacunes. Ils ont donc tout en main pour assurer leur progression. 
L'étudiant devrait avoir un sentiment de compétence plus important lorsque, parti de son propre niveau, avec ses propres prérequis, le dispositif le pousse à aller le plus loin possible. Le tuteur l'accompagne dans cette progression personnelle et soutient le travail d'autoévaluation de l'étudiant (bilan), qui lui permet d'objectiver les compétences réellement développées et le degré de maîtrise des contenus abordés.

Le dispositif favorise l'engagement et la persévérance des étudiants dans l'apprentissage de tâches complexes $^{[11]}$ : ils sont invités à exprimer leurs besoins d'apprentissage et les tuteurs et enseignants $\mathrm{y}$ répondent.

Les connaissances antérieures sont prises en compte : les étudiants sont amenés à décrire dans leur portfolio leurs connaissances, vécu et expériences antérieurs après analyse de la vignette clinique.

Les nouveaux apprentissages sont intégrés aux acquis antérieurs lorsque les étudiants révisent leur prise en charge après les enseignements théoriques reçus.

L'étudiant est orienté vers des objectifs pertinents ${ }^{[11]}$ qui lui sont communiqués clairement au début du dispositif grâce au guide d'accompagnement. Ces objectifs correspondent à des capacités professionnelles et sont adaptés aux besoins actuels des étudiants, tout en plaçant ces derniers face à des défis en termes d'apprentissage.

Ces objectifs sont en concordance avec l'évaluation $^{[11]}$. La grille d'indicateurs et de critères est communiquée très tôt aux étudiants. C'est sur cette base que ceux-ci reçoivent un feed-back au terme $\mathrm{du}$ dispositif par les enseignants responsables du cours.

Les étudiants ont eu l'occasion d'exprimer leurs émotions et leurs croyances, ce qui est essentiel dans ce domaine particulièrement sensible des soins palliatifs. Les étudiants peuvent en effet se trouver touchés, affectés, interpellés par l'histoire du patient et le vécu des professionnels. La motivation à apprendre est en effet influencée par les états émotionnels et les croyances ${ }^{[11]}$ des étudiants. Ces émotions doivent pouvoir être exprimées et travaillées, notamment grâce à une démarche réflexive, suscitée par le portfolio et le contact avec les tuteurs.

\section{Développer des capacités réflexives}

Un certain nombre de conditions sont mis en œuvre pour favoriser la transférabilité des connaissances et des stratégies d'apprentissage ${ }^{[5]}$ : la démarche suscitée est proche de l'activité professionnelle (analyse, réflexivité). La vignette clinique est également proche de la réalité professionnelle. L'étudiant est soutenu dans l'identification de ses forces, de ses progrès, de ses lacunes et des leviers à la remédiation de son action (canevas d'auto-évaluation, bilan des compétences et accompagnement des tuteurs). La transférabilité souffre toutefois d'interactions explicites avec le terrain. Il n'y a effectivement pas de possibilité actuellement d'établir de liens entre le cours et les lieux professionnels et donc des allers et retours conceptuels.

Il est reconnu qu'il est important de soutenir et d'accompagner la pratique réflexive des étudiants, dès le début des apprentissages si on veut favoriser l'adoption ultérieure de cette pratique ${ }^{[17]}$. Par leurs interactions à distance avec chaque étudiant, les tuteurs, médecins chevronnés, contribuent à développer la pratique réflexive des étudiants au moyen du portfolio individuel et des grilles d'auto-évaluation. Cette influence pourrait être plus marquée si il y avait plus de moments en présentiel.

La réflexivité n'est pas un processus évident; le plus souvent d'ailleurs, il est tacite chez les professionnels ${ }^{[17]}$. Dans le guide d'accompagnement, des questions sont proposées aux étudiants pour les aider à adopter une posture réflexive, à s'interroger. Des illustrations de réponses sont également suggérées afin d'illustrer des niveaux de réflexivité différents.

Les étudiants sont demandeurs d'une rétroaction sur le contenu et le processus de leur réflexion ${ }^{[17]}$ : ici, les tuteurs interagissent à chaque étape du dispositif avec chaque étudiant sur la pertinence des connaissances décrites, sur la profondeur et la pertinence de leur analyse, sur les scotomes éventuels, etc. Des rubriques du portfolio sont prévues pour le feed-back des tuteurs et les réactions de l'étudiant. C'est l'occasion pour l'étudiant d'examiner ses forces et faiblesses et de déterminer ses besoins d'apprentissage avec l'aide du tuteur. Les enseignants eux, évaluent le portfolio en fin de processus et transmettent leur analyse à l'étudiant. 
La réflexivité peut être un outil d'auto-évaluation encourageant les étudiants à améliorer leur jugement ${ }^{[17]}$. Grâce aux grilles d'autoévaluation, les étudiants sont invités à réguler leurs actions et à évoquer des perspectives d'apprentissage ${ }^{[8,9]}$. Les tuteurs les encouragent à identifier d'autres objectifs ou à approfondir leur réflexion.

La réflexivité devrait faire partie de la culture d'enseignement et d'apprentissage et devrait être valorisée et légitimée ${ }^{[17]}$. Dans le dispositif, la réflexivité des étudiants est effectivement évaluée grâce à une traduction en critères et indicateurs. Elle est explicitement prise en compte dans les grilles d'évaluation par objectifs et fait l'objet d'une remise de note. Par contre la réflexivité ne fait en aucune manière partie de la culture des études médicales. Il s'agit là d'un frein important au développement de cette capacité chez les étudiants qui, en avant-dernière année des études médicales de base, n'ont pas été encouragés explicitement à porter un regard réflexif sur leurs performances, leurs lacunes et la manière d'y remédier de façon proactive et autonome.

Des opérations cognitives de haut niveau ${ }^{[11]}$ sont suscitées et mises en œuvre par les étudiants tout au long du dispositif au moyen notamment des grilles d'auto-évaluations mensuelles dans lesquelles les étudiants sont amenés à prendre du recul par rapport à leurs compétences. Dans la taxonomie de Bloom, ces opérations cognitives correspondent à l'analyse (niveau 4) et à l'évaluation (niveau 6).

La pratique réflexive est cruciale en médecine car le métier de médecin, et plus particulièrement la pratique des soins palliatifs et de fin de vie, nécessite une réflexion importante sur ses actions et ses démarches éthiques. Il était donc important de proposer aux étudiants des activités réflexives pour un domaine qui fera précisément appel à la réflexivité dans l'exercice professionnel. On peut parler ici d'une forme d'isomorphisme ${ }^{[25]}$ dans la formation proposée aux étudiants, qui consiste à faire vivre aux étudiants des aptitudes, des capacités qu'ils seront invités à mettre en œuvre dans leur vie professionnelle.

Le rôle du tuteur s'est avéré crucial et très apprécié des étudiants. La supervision de l'étudiant est considérée comme indispensable dans une approche centrée sur le portfolio, notamment lorsque celui-ci est de type électronique. L'étudiant doit en effet être assisté dans sa démarche d'apprentissage et d'autoévaluation, condition nécessaire d'une pratique réflexive qui n'est pas spontanée ${ }^{[23,24]}$

\section{Les faiblesses du dispositif}

\section{Les problèmes techniques}

Une lenteur d'accès au site web accueillant les portfolios électroniques, des problèmes de mots de passe et de sauvegarde des données ont démotivé considérablement les tuteurs et les étudiants. La communication entre eux s'en est trouvée limitée et parfois entravée.

La modalité électronique a été choisie pour permettre aux étudiants et aux tuteurs d'interragir à distance à des moments qui leur conviennent le mieux (anywhere, anytime). Des contraintes horaires empêchaient d'autres modalités de rencontre et d'interactions en présentiel entre tuteurs et étudiants.

Spontanément, on pourrait croire qu'un outil électronique permet un gain de temps et d'énergie aux participants. Or, Deketelaere ${ }^{[21]}$ montre que l'investissement qu'implique le tutorat au moyen d'un portfolio électronique peut être plus consommateur de temps encore qu'une rencontre individuelle. L'aspect électronique de l'outil implique qu'il faille une préparation et une réflexion spécifiques avant de mettre en œuvre un tel dispositif ${ }^{[21]}$.

\section{L'enchâssement dans la pratique professionnelle}

Le cours de médecine palliative et de fin de vie est un cours introductif. Il a pour but de susciter chez l'étudiant une première réflexion en la matière. Toutefois, l'idéal serait d'intégrer la dimension interactive du «triptyque socio-constructiviste ${ }^{[6]}$ en faisant en sorte que les étudiants soient intégrés à un stage où est abordée la médecine palliative, tout au long du dispositif décrit ici. Ceci relève cependant encore de la gageure. 


\section{La formation des tuteurs}

Il ne suffit certainement pas de désigner un tuteur pour que des bénéfices soient effectifs (impact sur l'apprentissage des étudiants). Le tuteur doit être formé et accompagné lui aussi. Des types d'intervention doivent être définis et explicités ${ }^{[23]}$. C'est en ce sens que le dispositif devra être amélioré dans les années à venir.

\section{La démarche réflexive}

Malgré le dispositif d'accompagnement basé sur le tutorat et l'élaboration d'un guide de rédaction fournissant aux étudiants des consignes précises, certains éprouvent des difficultés à poser un regard réflexif sur leurs apprentissages et compétences ${ }^{[26]}$. Dès lors, il nous semble important d'ajuster notre dispositif en exemplifiant davantage les démarches demandées dans notre guide ainsi que les critères d'évaluation. De plus, les étudiants qui le souhaitent pourront bénéficier d'entretiens de régulation axés sur les démarches réflexives à mettre en œuvre.

\section{Conclusions}

Les auteurs avaient pour objectif de répondre à la problématique de la désertification de l'amphithéâtre et du manque d'intérêt des étudiants pour le cours de médecine palliative et de fin de vie Les enseignants se sont appuyés sur le paradigme socio-constructiviste et sur le développement d'une pratique réflexive pour construire le nouveau dispositif pédagogique. Celui-ci a été mis en place comme prévu mais l'évaluation de ses impacts effectifs sur les apprentissages reste à documenter.

\section{Remerciements}

Les auteurs tiennent à remercier vivement les tuteurs bénévoles sans qui le dispositif n' aurait pu avoir lieu. Ils remercient également les étudiants qui se sont prêtés à cette première expérience.

\section{Contributions}

Didier Giet et Jean-François Brichant ont conçu le dispositif avec le soutien pédagogique de Valérie Massart et Mélanie Deum. Valérie Massart a écrit le manuscrit en collaboration avec Didier Giet et Mélanie Deum. Jean-François Brichant a assuré une relecture précise de l'article.

\section{Références}

1. Leclercq D. Méthodes de formation et théories de l'apprentissage. Liège : Université de Liège, Les Éditions de l'ULg, 2003.

2. Ausubel D. The Psychology of Meaningful Verbal Learning. New York: Grune \& Stratton, 1963.

3. Vygotsky L. Pensées et langage. Paris : La Dispute, 1934/1997.

4. Bandura A. Social learning theory. Englewood Cliffs (NJ): Prentice-Hall, 1977.

5. Jouquan J, Bail Ph. À quoi s'engage-t-on en basculant du paradigme d'enseignement vers le paradigme d'apprentissage ? Pédagogie Médicale 2003;4:16375.

6. Jonnaert $\mathrm{Ph}$, Vander Borght C. Créer des conditions d'apprentissage. Un cadre de référence socioconstructiviste pour une formation didactique des enseignants. Perspectives en éducation et formation. Bruxelles : Ed. De Boeck, 2009.

7. Deum M, Vanhulle S. Portfolio et démarches socioréflexives en enseignement et formation. Genève : Cahier de la section des sciences de l'éducation $\mathrm{n}^{\circ}$ 116. Pratiques Théorie, 2008.

8. Allal L. Impliquer l'apprenant dans le processus d'évaluation : promesses et pièges de l'autoévaluation, in Depover C., Noël B. (Éds) L'évaluation des compétences et des processus cognitifs. Modèles, pratiques et contextes, Bruxelles: De Boeck \& Larcier, 1999 ; pp. 35-56.

9. Boud D. Enhancing learning through Self Assessment. London: Kogan Page, 1995.

10. De La Cruz M.A, Gomez E, Martinez M.F. Autoévaluation du professeur en tant qu'une voie pour 
l'amélioration de la qualité de l'enseignement universitaire, in Boxus E. et al. (Éds) Stratégies et médias pédagogiques pour l'Apprentissage et l'Évaluation dans l'Enseignement Supérieur, Actes du $15^{\mathrm{e}}$ colloque de l'Association Internationale de Pédagogie Universitaire (AIPU), Université de Liège, 1997 : 171-81.

11. American Psychological Association (APA). Learnercentered psychological principles: a framework for school redesign and reform (1997 revision). Washington (DC) : APA Presidential Task Force on Psychology in Education. [on-line] Disponible sur : http://www.apa.org/ed/governance/bea/learnercentered.pdf (consulté en mai 2011).

12. Perrenoud $\mathrm{Ph}$. Développer la pratique réflexive. Dans le métier d'enseignant. Paris : ESF éditeur, 2001.

13. Schön DA. The Reflective Practitionner. New York : Basic Books, 1983. Traduction Française : Le praticien réflexif. À la recherche du savoir cache dans l'agir professionnel. Montréal : Éditions Logiques, 1994.

14. Hubert, S., Bosmans C., Denis B. Différents niveaux de problèmes : Impact du cours d'Éducation par la Technologie : Mise au point d'outils d'évaluation des compétences transversales, Rapport de recherche, Service de Technologie de l'Éducation, Université de Liège, 1998.

15. Jouquan J. À quelles conditions le portfolio peut-il tenir ses promesses comme soutien à l'apprentissage en profondeur et à la pratique réflexive ? Colloque sur le portfolio en enseignement supérieur. Comment accompagner l'étudiant dans sa démarche ? 6 novembre 2009 ; Centre d'études et de formation en enseignement supérieur (CEFES), Université de Montréal, Canada ; 2009.

16. Gombert JE. Le développement métalinguistique. Paris : PUF, 1990.

17. Mann K, Gordon J, MacLeod A. Reflection and reflective practice in health professions education: a systematic review. Adv in Health Sci Educ 2009;14:595-621.

18. Viau R. La motivation en contexte scolaire. Bruxelles: De Boeck, 2007.

19. Walker, D. Writing in reflexion. In: Keogh R, Walker D. Reflection: turning experience into learning. London: Logan, 1985:52-68.

20. Levine R, Kern D, Wright S. The impact of prompted narrative writing during internship on reflective practice: a qualitative study. Adv Health Sci Educ 2008; 13: 723-33.

21. Deketelaere A, Degryse, De Munter A, De Leyn P. Twelve tips for successful e-tutoring using electronic portfolios. Med Teach 2009;31:497-501.

22. Tardif J. Intégrer les nouvelles technologies de l'information. Quel cadre pédagogique ? Paris : ESF, 1998.

23. Depover Ch, De Lievre B, Peraya D, Quintin JJ, Jaillet A. Le tutorat en formation à distance. Perspectives en éducation et formation. Bruxelles : Ed. De Boeck, 2011.

24. Naccache N, Samson L, Jouquan J. Le portfolio en éducation des sciences de la santé: un outil d'apprentissage, de développement professionnel et d'évaluation. Pédagogie Médicale 2006;7:110-27.

25. Guillaume F-F, Xhonneux M. Faire et faire faire. Le Principe d'isomorphisme dans la formation des enseignants de sciences sociales, CIFEN, Centre Interfacultaire de formation des enseignants. Liège : Université de Liège, Bulletin n²2, 2007 : 73-77.

26. Jans V. Favoriser les processus réflexifs en éducation, module 8 de la formation Form@ @icef. Liège : Université de Liège, 2010.

Correspondance et offprints: Didier Giet, Université de Liège, Département de médecine générale (DUMG). Institut de Formation et de Recherche en Enseignement Supérieur (IFRES). 1 avenue de l'Hôpital, B23, 4000 Liège, Belgique.

Mailto : D.Giet@ulg.ac.be 